\title{
ANALYSE QUANTITATIVE DE LA QUALITÉ DES IMAGES OBTENUES PAR UN SONAR À OUVERTURE SYNTHÉTIQUE
}

\author{
J. CHATILLON, M.E. ZAKHARIA et M.E. BOUHIER* \\ Institut de Chimie et de Physique Industrielles de Lyon, I.C.P.I. Lyon, Laboratoire d'Acoustique \\ Systèmes et Signaux SOnar (L.A.S.S.SO.), 31 Place Bellecour, F-69288 Lyon cedex 02, France \\ *Institut Français de Recherche pour l'Exploitation de la Mer, IFREMER, Centre de Toulon, Zone \\ Portuaire de Brégaillon, BP. 330, F-83507 La Seyne sur Mer, France
}

\begin{abstract}
Résumé: L'évaluation des performances d'un sonar à ouverture synthétique pose le problème de la quantification de la qualité des images obtenues, qui est liée, entre autres, à l'uniformité de la trajectoire de la plate-forme sonar. Dans le cas où cette trajectoire est perturbee, nous allons chercher à quantifier la dégradation d' images obtenues par simulation. Le critère retenu est une différence normée entre l'image parfaite et l'image dégradée. Son étude est effectuée au moyen de différentes mires acoustiques dans plusieurs cas de navigation. Le but de l'étude est ainsi de disposer d'un critère de qualité unique permettant d'analyser de façon statistique l'influence des perturbations aléatoires.

Abstract: Performance evaluation of a synthetic aperture sonar leads to the estimation of the quality of obtained images which is closely related to the sonar platform trajectory. In case of navigational inaccuracies, the degradation of this quality needs to be quantified. The proposed criterion is the normalized difference between a perfect image and damaged ones. Its study (by means of simulations) is achieved using several typical target configurations for different navigation cases. The aim of this work is therefore to define one simple quality criterion which will enable the influence of random perturbations to be statistically analysed.
\end{abstract}

\section{Introduction.}

Dans le cadre de l'imagerie acoustique par sonar latéral actif, nous envisageons d'utiliser une antenne synthétique qui peut présenter divers avantages opérationnels: réduction de l'encombrement du système remorqué, meilleure résolution azimutale indépendante de la distance, possibilité de fonctionnement en basse-fréquence. Des travaux antérieurs [1],[2] ont montré l'intérêt d'utiliser des signaux large-bande en émission: lélargissement de la bande permet en effet d'augmenter la résolution latérale (après filtrage adapté) et la résolution azimutale (par lissage des fréquences spatiales des lobes latéraux de l'antenne) des images. Il ouvre aussi des perspectives d'investigation de la classification des échos et/ou du sous-échantillonnage spatial [3]. Les perturbations de la trajectoire de l'antenne remorquée, l'effet Doppler ou les problèmes de propagation peuvent dégrader la qualité des images obtenues par synthèse d'antenne. Pour quantifier ces dégradations, nous simulons des acquisitions de données sonar pour diverses configurations de cibles dans le cas d'une trajectoire quelconque. Le calcul d'antenne synthétique est ensuite effectué sur ces données en supposant la trajectoire rectiligne uniforme. Nous comparons alors qualitativement et quantitativement les images obtenues dans le but de connaître les limites acceptables de ces perturbations et de mieux comprendre les phénomènes mis en jeu. Le but de cette étude est de trouver un critère objectif permettant d'intégrer au mieux toute la notion subjective de qualité d'image. Un tel critère pourra alors être utilisé pour une étude systématique des perturbations aléatoires de trajectoire.

\section{Approche classique: résolution azimutale.}

Les approches classiques de qualité d'image acoustique s'expriment souvent en terme de résolution (ou de perte de résolution). Ces méthodes, inspirées de l'optique, se résument à des mesures de largeur du lobe principal de la réponse en azimut d'une configuration comportant une ou deux cibles ponctuelles. Ces approches que l'on qualifiera de "séparatives" sont largement utilisées en optique et en imagerie acoustique. La plus simple consiste à mesurer la largeur du lobe principal (à -3dB par exemple) de la réponse en azimut d'une configuration comportant une cible ponctuelle (figure 1). Si son mérite est d'être simple à mettre en œuvre et d'être universelle, sa limitation est évidente puisqu'elle ne tient pas compte des lobes secondaires de la 
réponse alors que leur influence peut être prépondérante. La mesure d'un index de directivitê, qui consiste à intégrer cette réponse sur les angles d'observation, permet cependant de tenir compte de ces lobes secondaires ou du moins de leur niveau moyen autour de l'angle d'observation.

Pour mesurer efficacement la résolution en azimut de deux cibles ponctuelles, il faut en fait systématiquement utiliser le critère de Rayleigh: deux cibles ponctuelles, situées à la même distance et à des azimuts différents, sont résolues si leurs réponses sont séparées à un niveau fixé, classiquement $-3 \mathrm{~dB}$ (figure 2 ).

Si ces critères sont très utiles pour comparer les performances obtenues sans perturbation pour diverses configurations sonar, ils deviennent cependant insuffisants dès que les perturbations de trajectoire sont importantes. Celles-ci peuvent en effet induire des effets difficiles à représenter sous forme de grandeurs mono-dimensionnelles (figures 3 et 4): migrations des cibles, apparition d'échos fantômes, diminution du rapport signal à bruit, etc...

La solution proposée est d'utiliser des cibles étalons (mires acoustiques) similaires à celles largement utilisées par les typographes ou opticiens dans le but de définir un quantificateur, le plus objectif possible, de la qualité des images dégradées.

Comme les deux résolutions (azimutale mais aussi latérale) sont mises en jeu lors de dégradations de l'image obtenue, nous définirons une méthode de mesure globale de limage.

\section{Critère d'erreur et mires acoustiques associées.}

Pour caractêriser la qualité d'une image synthétique, certains auteurs [4] proposent de calculer une distance entre limage parfaite et une image dégradée comportant des cibles ponctuelles. Cette distance est normée par des coefficients de pondération tenant compte de la configuration sonar étudiée: longueur d'antenne, longueur d'onde, position réelle des cibles, etc.... Dans le cas de signaux d'émission large bande, la normalisation vis à vis de la longueur d'onde n'est pas unique. Cette méthode s'applique difficilement à des cibles étendues. Le choix des coefficients de pondération, choix nécessaire pour rendre le résultat indépendant de la configuration sonar, est forcément discutable.

Une approche indépendante de la configuration sonar est l'utilisation d'une distance très classique entre l'image parfaite $\mathrm{I}_{\mathrm{ref}}(\mathrm{x}, \mathrm{y})$ et l'image dégradée $\mathrm{I}_{\mathrm{a}}(\mathrm{x}, \mathrm{y})$ égale à l'erreur quadratique moyenne $\varepsilon_{\mathrm{d}}$ telle que:

$$
\varepsilon_{d^{2}}=\frac{\sum_{i=1}^{m}\left(I_{r e f}\left(x_{i}, y_{i}\right)-I_{a}\left(x_{i}, y_{i}\right)\right)^{2}}{\sum_{i=1}^{m}\left(I_{r e f}\left(x_{i}, y_{i}\right)\right)^{2}}
$$

les images étant de dimension m par m pixels.

Ce quantificateur simple a le mérite de tenir compte de toute l'information présente dans l'image tout en pouvant être appliqué à tout type de cibles. I reste cependant a priori dépendant du type de mire acoustique utilisée.

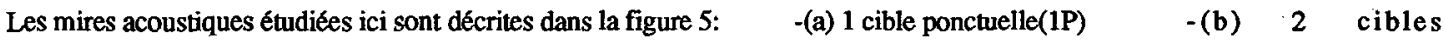
ponctuelles à la même distance et séparées en azimut(2P) $\quad$-(c) 3 cibles ponctuelles: configuration (b) plus une cible ponctuelle supplémentaire séparée en distance et placée à l'azimut moyen (3P) -(d) 4 cibles étendues en azimut séparées 2 par 2 en azimut et en distance(4E).

Les distances inter-cibles ont été choisies de manière à ce que les cibles soient en limite de résolution dans le cas où la trajectoire est parfaite.

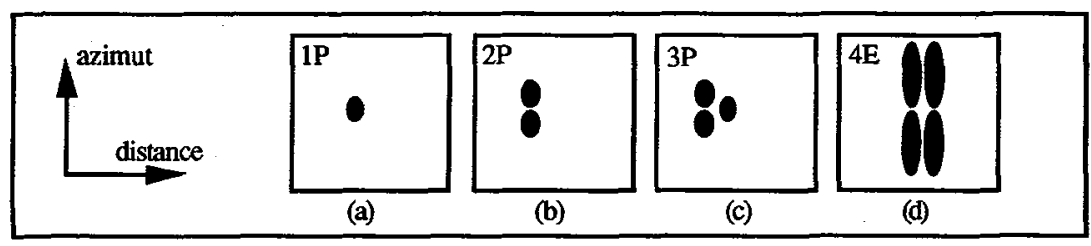

Figure 5: Mires acoustiques utilisées 


\section{Résultats.}

Nous calculons d'abord, pour chacune des 4 mires, les images de référence associées en simulant les acquisitions sonar pour une trajectoire de la plate-forme rectiligne et uniforme. Nous étudions ensuite 3 lacets périodiques différents. Leur période TL est comparée à la longueur de l'antenne synthétique $\mathrm{L}_{\mathbf{S a}}$, leurs amplitudes $A_{L}$ sont comparées à la longueur d'onde $\lambda_{0}$ pour la fréquence centrale du signal d'émission:

$$
\begin{array}{lll}
\text { - Ier lacet: } & \mathrm{T}_{\mathrm{L} 1}=\mathrm{L}_{\mathrm{sa}} / 15 & \text { (noté } \mathrm{HF} \text { pour haute fréquence) } \\
\text { - 2ème lacet: } & \mathrm{T}_{\mathrm{L} 2}=\mathrm{L}_{\mathrm{sa}} / 3 & \text { (noté MF pour moyenne fréquence) } \\
\text { - 3ème lacet: } & \mathrm{T}_{\mathrm{L} 3}=\mathrm{L}_{\mathrm{sa}} / 0.8 & \text { (noté } \mathrm{BF} \text { pour basse fréquence) }
\end{array}
$$

Leurs amplitudes sont définies par $A_{L}=a . \lambda_{0} / 8(0,1<a<10) . \lambda_{0} / 8$ est la limite utilisée dans la littérature [5] en deçà de laquelle on obtient théoriquement toujours, pour l'ouverture synthétique, une résolution azimutale égale à la moitié de la longueur de l'antenne physique.

Les simulations réalisées [1] ont montré de façon qualitative que l'effet apparent du lacet périodique dépendait de la période de ce dernier: un lacet BF induit plutôt des pertes de contraste et des migrations (figure 3) tandis qu'un lacet HF fait apparaître des échos fantômes (figure 4).

Les figures 6 et 7 montrent des exemples typiques de variation de l'erreur quadratique $\varepsilon_{d}(e n \%)$ en fonction de $\log (a)$. D'une façon plus générale, l'analyse des résultats conduit aux remarques suivantes:

- à mire acoustique fixée: pour des petites perturbations de trajectoire $\left(A_{L}<\lambda_{0} / 8\right)$, l'ordre de grandeur de l'erreur mesurée est le même quelle que soit la période du lacet. Pour des perturbations plus importantes, les courbes divergent (figure 6).

- à lacet fixé: le niveau de l'erreur dépend toujours du choix de la mire. D'une façon générale, l'erreur associée aux cibles ponctuelles est toujours plus importante que celle associée aux cibles étendues, ces dernières minimisant l'erreur grâce à un lissage spatial (ce ne sont pas, contrairement aux cibles ponctuelles, des cibles omnidirectionnelles: figure 7).

\section{Conclusion.}

La difficulté de la description de la qualité d'une image sonar obtenue par synthèse d'ouverture nous a conduit à mettre au point une technique d'évaluation similaire à celle utilisée par les typographes et les opticiens. L'association de ces mires à un critère d'erreur quadratique moyenne permet d'avoir une approche à la fois simple et objective du problème posé.

Les essais réalisés ont montré l'intérêt d'une telle approche. Le choix de la mire acoustique standard reste cependant délicat et dépend essentiellement de l'application envisagée (cartographie, bathymétrie, reconnaissance d'objets...).

Ce choix ainsi que la quantification de l'erreur associée devront être corrélés à des expertises d'opérateurs qualifiés afin de pouvoir établir un seuil définissant une qualité d'image acceptable.

L'établissement de ce seuil nous permetura alors d'étudier les perturbations aléatoires de la trajectoire de la plate-forme sonar qui nécessitent un grand nombre de simulations et une étude statistique de la qualité d'image.

\section{Références.}

[1] J. CHATILLON, M.E. BOUHIER and M.E. ZAKHARIA: "Synthetic aperture sonar: wide band vs narrow band." U.D.T. Conference, Paris, 1991, pp. 1220-1225.

[2] M.E. ZAKHARIA, J, CHATILLON and M.E. BOUHIER: "Synthetic aperture sonar : a wide band approach." I.E.E.E. Ultrasonics Symposium, Honolulu Hawaii, U.S.A., December 1990, pp 1133-1136.

[3] P. de HEERING: "Alternate schemes in various sonar processing." IEEE J. Oceanic Eng., vol. oe-9, 4, October 1984, pp 277-280.

[4] D.G. CHECKETTS and B.V. SMITH: "Analysis of the effects of platform motion errors upon various sonar." Proc. I.O.A., 1986, Vol 8, N³, pp 135-143.

[5] L.J. CUTRONA: "Comparison of sonar system performance achievable using synthetic aperture techniques with the performance achievable by more conventional means." J. Acous. Soc. Am., August 1975, Vol. 58, N², pp $336-348$.

\section{Remerciements.}

Cette étude est supportée par la Communauté Européenne, DG XII, (programme MA.S.T., projet AC.I.D.). 


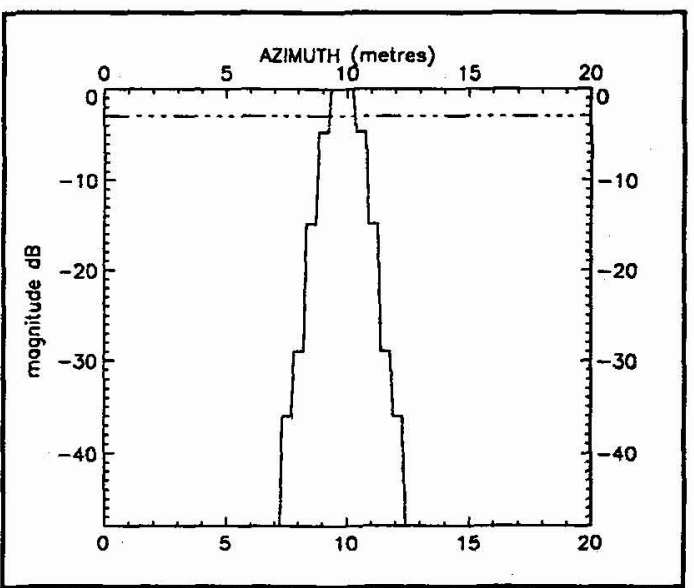

Figure 1: Mesure de largeur du lobe principal de la réponse en azimut d'une seule cible ponctuelle.

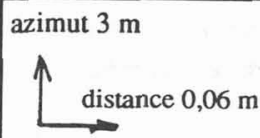

position

réelle des 2

cibles

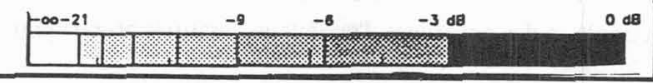

Figure 3: Image de 2 cibles ponctuelles dégradée par du lacet BF: migrations, atténuation.

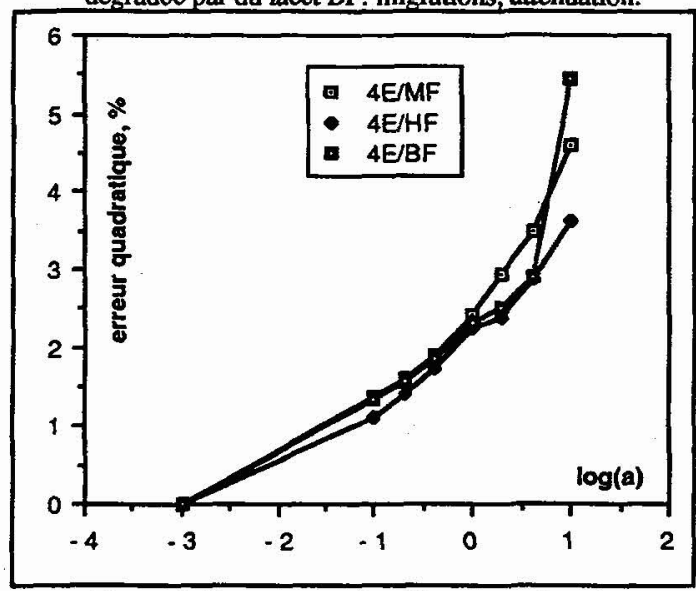

Figure 6: Erreurs, pour les 3 lacets, en fonction de l'amplitude de la perturbation, mire $4 \mathrm{E}$.

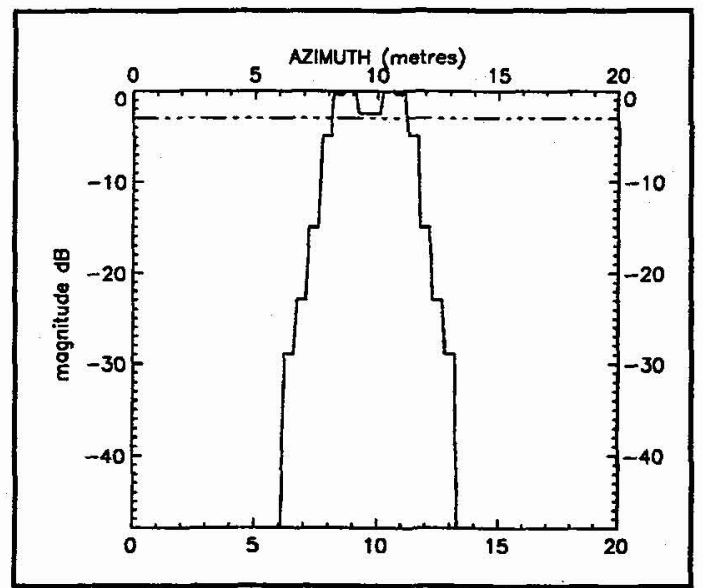

Figure 2: Critère de Rayleigh appliqué à 2 cibles ponctuelles.

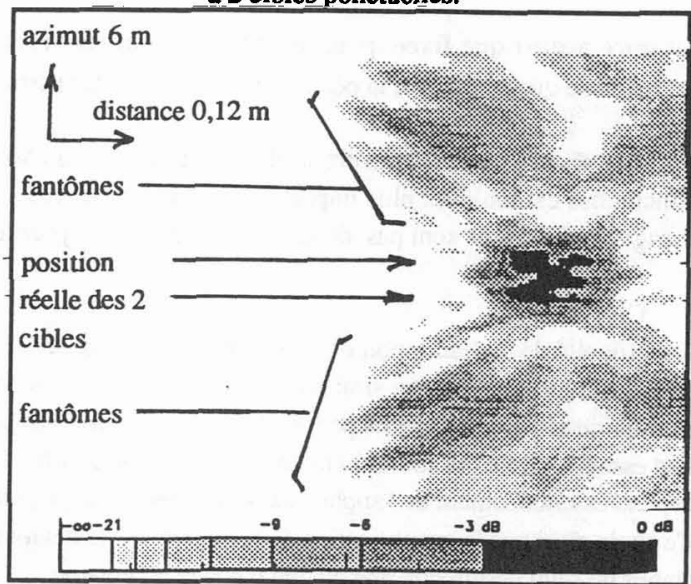

Figure 4: Image de 2 cibles ponctuelles dégradke par du lacet HF: fantômes.

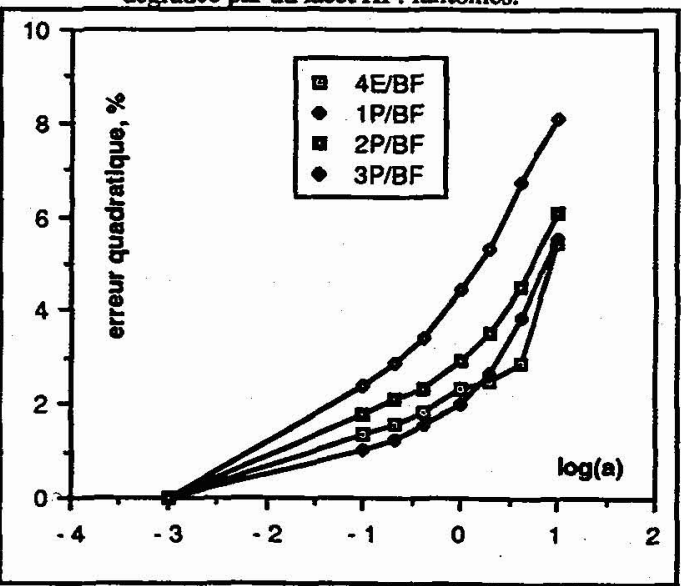

Figure 7: Erreurs, pour les 4 mires, en fonction de l'amplitude de la perturbation, lacet BF. 